\title{
Apendicectomía laparoscópica en pacientes con apendicitis aguda complicada con base apendicular comprometida: estudio de cohorte retrospectivo
}

\author{
Laparoscopic appendectomy in complicated appendicitis with compromised appendix \\ base: a retrospective cohort study \\ Karina Flores-Marín, Andrés Rodríguez-Parra, Mario Trejo-Ávila*, Eduardo Cárdenas-Lailson, Roberto \\ Delano-Alonso, Carlos Valenzuela-Salazar, Jesus Herrera-Esquivel y Mucio Moreno-Portillo \\ Departamento de Cirugía General y Endoscópica, Hospital General Dr. Manuel Gea González, Ciudad de México, México
}

\section{Resumen}

Antecedentes: Los pacientes con base apendicular comprometida constituyen un subgrupo de pacientes con apendicitis complicada y existe poca información al respecto. Objetivo: Conocer la frecuencia de fístulas y fugas fecales en pacientes con apendicitis complicada con base apendicular comprometida. Método: Se trata de un estudio observacional, retrospectivo y transversal de pacientes operados de apendicectomía laparoscópica con base apendicular comprometida. Resultados: De 2015 a 2019 se encontraron 158 casos de apendicitis complicada, de los cuales 54 tenían la base apendicular comprometida. Hubo predominio de varones (66.7\%) y la edad media fue de 38.7 años. En el $57.4 \%$ de los casos se realizó un nudo simple y en el $42.6 \%$ un punto transfictivo con invaginación del muñón. En relación con las complicaciones, el $16.7 \%$ desarrollaron abscesos intraabdominales, el 7.4\% íleo y el 7.4\% infección de herida. Hubo un paciente con fuga del muñón y un paciente con fístula cecal. El tiempo medio de estancia hospitalaria fue de 5.4 días (rango: 1-20). Se realizaron cinco reintervenciones: cuatro para drenaje de absceso intraabdominal y una por fuga del muñón. Conclusiones: En los pacientes con base apendicular comprometida, el manejo laparoscópico con ligadura simple o con punto transfictivo resulta en una baja frecuencia de fuga y fístula del muñón apendicular.

Palabras clave: Apendicitis aguda. Apendicectomía laparoscópica. Apendicitis complicada. Cierre de muñón apendicular.

\begin{abstract}
Background: Patients with compromised appendix base constitute a subgroup of patients with complicated appendicitis, and there is few available information. Objective: To study the frequency of stump leaks and fistulae in patients with complicated appendicitis with compromised stump. Method: This is an observational, retrospective study of patients that underwent laparoscopic appendectomy with compromised appendix stump. Results: From 2015 to 2019, 158 patients with complicated appendicitis were operated, of them 54 had compromised base or stump. There were $66.7 \%$ men, with a mean age of 38.7 years. For stump closure, a simple knot was employed in $57.4 \%$, and in $42.6 \%$ an invaginated suture was employed. Regarding complications, $16.7 \%$ developed intraabdominal abscess, $7.4 \%$ ileus and $7.4 \%$ had wound infection. We found one stump leak
\end{abstract}

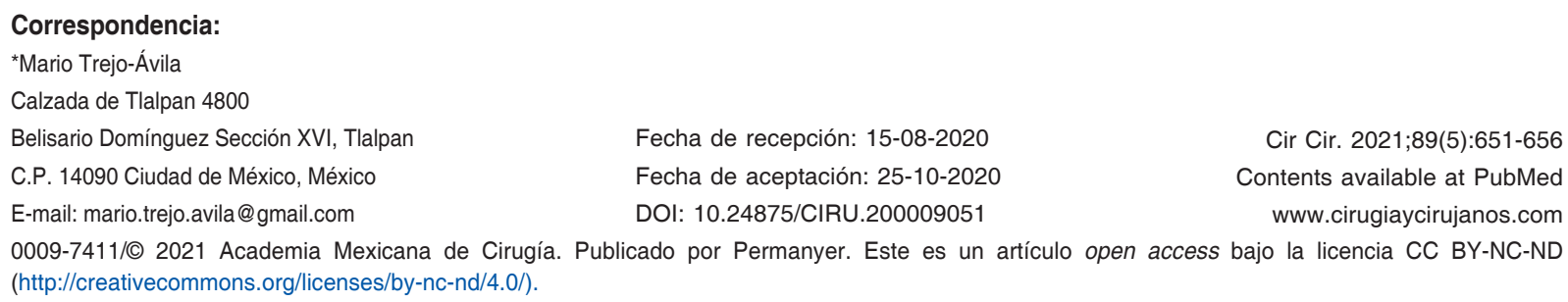

Cir Cir. 2021;89(5):651-656 Contents available at PubMed www.cirugiaycirujanos.com 0009-7411/C 2021 Academia Mexicana de Cirugía. Publicado por Permanyer. Este es un artículo open access bajo la licencia CC BY-NC-ND (http://creativecommons.org/licenses/by-nc-nd/4.0/). 
and one stump fistula. The mean length of stay was 5.4 days (range: 1-20). There were 5 reoperations, 4 for abscess drainage and 1 for stump leak. Conclusions: Patients with acute complicated appendicitis with compromised appendicular base, laparoscopic surgery either with simple knot or with invaginated suture resulted in low frequency of stump leaks and fistula.

Key words: Acute appendicitis. Laparoscopic appendectomy. Complicated appendicitis. Appendix stump closure.

\section{Introducción}

La apendicitis aguda, descrita por primera vez por Heber Fitz en 1886, actualmente es la causa mas común de dolor abdominal agudo1. La incidencia general de apendicitis aguda varía entre 76 y 227 casos por 100.000 habitantes por año². Se ha reportado que el riesgo general de padecer apendicitis aguda se encuentra entre el $6 \%$ y el $16 \%$. Esta condición afecta a todos los grupos de edad, con una mayor incidencia en la segunda década de la vida ${ }^{3}$.

El diagnóstico de apendicitis aguda complicada se realiza por el aspecto macroscópico durante el transoperatorio o por estudio histopatológico, y se define como un apéndice que se encuentra necrosado o perforado, o con un absceso pélvico y peritonitis generalizada ${ }^{2-6}$.

El tratamiento estándar continúa siendo la apendicectomía, sea con abordaje abierto o laparoscópico. La apendicectomía laparoscópica se describió por primera vez en $1983^{5}$. Es una técnica quirúrgica bien descrita y ha ganado amplia aceptación porque ofrece menos infecciones de herida quirúrgica, menor dolor posoperatorio, una recuperación rápida y un retorno más temprano al trabajo, en comparación con la cirugía abierta ${ }^{6}$.

Una técnica segura de cierre del muñón es un aspecto clave cuando se trata de una apendicitis aguda complicada, ya que la inflamación local y la infección pueden provocar complicaciones como fuga fecal, fístulas fecales o infección de sitio quirúrgico órgano-espacio (absceso intraabdominal). En todo el mundo se utilizan diferentes métodos de cierre del muñón apendicular, que pueden dividirse en ligaduras tradicionales (como las suturas intracorpóreas o extracorpóreas o endo-loops) y ligaduras con dispositivos mecánicos (engrapadoras, endoclips o dispositivos electrotérmicos) $)^{6-8}$. En nuestro país, la disponibilidad de dispositivos mecánicos es limitada, por lo que los métodos de ligadura tradicional constituyen una herramienta básica para el manejo del muñón apendicular y son parte fundamental en las destrezas del cirujano general. Las ligaduras tradicionales son métodos muy económicos, pero suelen prolongar el tiempo quirúrgico en comparación con las técnicas automáticas (grapas) ${ }^{6-9}$. La formación de fístulas fecales después de la apendicectomía, aunque es una complicación poco frecuente, se asocia con una morbilidad significativa. Las fugas del muñón apendicular representan la principal causa de desarrollo de fístulas fecales ${ }^{10}$. Existen muy pocos estudios en la literatura médica, y ninguno en nuestro país, que hayan descrito la frecuencia de complicaciones específicas, como fístulas y fugas fecales, en pacientes con base apendicular comprometida.

Por lo tanto, el objetivo del presente estudio es conocer la frecuencia de fugas del muñón apendicular y de fístulas fecales en pacientes con apendicitis aguda complicada con base del apéndice comprometida operados con cirugía laparoscópica en nuestro hospital.

\section{Método}

Se realizó un estudio observacional, descriptivo, retrospectivo y transversal, de pacientes con apendicitis aguda complicada con base del apéndice comprometida que se operaron con cirugía laparoscópica en el Hospital General Dr. Manuel Gea González, en Ciudad de México. El estudio se realizó en el periodo de enero de 2015 a diciembre de 2019 a partir de la base de datos mantenida de forma prospectiva de apendicectomías laparoscópicas, así como mediante la búsqueda de información en los expedientes clínicos. Los datos de los pacientes se mantuvieron confidenciales.

El presente trabajo se realizó de acuerdo con las recomendaciones STROBE (STrengthening the Reporting of OBservational studies in Epidemiology) ${ }^{11}$ para el reporte de estudios observacionales. El estudio fue aprobado por el Comité de Ética e Investigación Biomédica del hospital (registro 04-82-2019).

Se incluyeron todos los pacientes con apendicitis aguda complicada con la base del apéndice comprometida mayores de 18 años, que fueron operados con cirugía laparoscópica durante el periodo mencionado, y sin distinción de sexo. Se excluyeron aquellos pacientes cuyos expedientes no se encontraron o no 
tuvieron la información completa necesaria para el estudio.

El objetivo primario fue conocer la frecuencia de fugas y fístulas fecales en pacientes con apendicitis aguda con base apendicular comprometida. Como objetivos secundarios describimos la frecuencia de otras complicaciones posoperatorias relacionadas con la apendicitis complicada, como infección del sitio quirúrgico superficial o profundo y de órgano/espacio (absceso intraabdominal), íleo posoperatorio, reintervención y muerte.

Se consideraron apendicitis aguda complicada aquellos casos en que se encontró necrosis (gangrena) 0 perforación del apéndice con o sin peritonitis local o generalizada durante la laparoscopía diagnóstica' ${ }^{12}$. A su vez, se corroboró el diagnóstico de apendicitis complicada mediante la revisión del reporte de histopatología, en donde se confirmó la presencia de necrosis o perforación apendicular en la porción de la base del apéndice. En cuanto a la definición de base apendicular, se consideró como aquella porción anatómica inicial del apéndice en su unión al ciego (tercio proximal del apéndice), en el punto donde confluyen las tenias del colon, e incluye la transición entre la parte final del ciego y el inicio del apéndice cecal ${ }^{8}$. La base apendicular se consideró comprometida cuando se encontró isquemia, necrosis o perforación, en relación con apendicitis aguda complicada, de dicha porción anatómica, descrita tanto en la nota posoperatoria del cirujano como en el reporte de histopatología. Se incluyeron como base apendicular comprometida aquellos reportes quirúrgicos en los que se hacía referencia a perforación o necrosis de la base apendicular, el tercio proximal del apéndice, el muñón apendicular y la porción de transición entre el ciego y el inicio del apéndice. Los hallazgos quirúrgicos de base apendicular comprometida y las variables transoperatorias se obtuvieron de los reportes posquirúrgicos redactados tras la apendicectomía laparoscópica por el cirujano a cargo del caso. Los hallazgos patológicos se obtuvieron de los reportes del departamento de patología, tanto macroscópicos como microscópicos.

Se definieron como fuga del muñón aquellos casos en los que hubo salida de material intestinal o fecal a través del muñón apendicular, ocasionando una fuga a la cavidad abdominal o la formación de una colección, así como sepsis abdominal. El diagnóstico de fuga se realizó por el aspecto del gasto en el drenaje o por tomografía de abdomen contrastada con salida de contraste a través del muñón en caso de no tener drenaje, durante el posoperatorio temprano.
Las fístulas del muñón apendicular o del ciego generalmente requieren más tiempo para desarrollarse (posoperatorio tardío) y deben tener un trayecto epitelizado entre el ciego y la piel. El gasto fecal se presenta a través del drenaje 0 , una vez retirado este, del orificio donde se encontraba. Se diagnosticaron con tomografía con contraste oral o mediante fistulografía por tomografía.

El procedimiento quirúrgico consistió en cirugía laparoscópica con técnica estandarizada en nuestro centro académico. El procedimiento se realiza con tres puertos (uno óptico y dos de trabajo). La cirugía comienza con laparoscopía diagnóstica e identificación del apéndice. A continuación se llevan a cabo la disección del mesoapéndice y la ligadura simple con sutura monofilamento, y la base apendicular con ligadura simple o con punto transfictivo e invaginante. Posteriormente se realiza aspirado de colecciones purulentas. La colocación de un drenaje (en general tipo Jackson-Pratt) es a consideración del cirujano tratante. Todas las cirugías son realizadas o supervisadas por un cirujano adscrito certificado.

Para la descripción de la cohorte de pacientes incluidos se incluyeron variables sociodemográficas, tales como edad, sexo, IMC, comorbilidad y riesgo anestésico según la ASA (American Society of Anesthesiology). A su vez, se incluyeron resultados transoperatorios y posoperatorios: tiempo quirúrgico, sangrado transoperatorio, tipo de manejo del muñón apendicular (sutura, endo-loop), conversión a cirugía abierta y tiempo de estancia hospitalaria. También se analizó la frecuencia de complicaciones posoperatorias, incluidas la fuga del muñón apendicular y la formación de fístula cecal, y el resto de las complicaciones (objetivo secundario), que se clasificaron de acuerdo con la clasificación de Clavien-Dindo ${ }^{13}$.

El periodo de recolección y seguimiento de datos incluyó hasta los 6 meses posoperatorios para el objetivo primario del estudio (fugas y fístulas del muñón) y los 90 días posoperatorios para las variables secundarias.

Los datos obtenidos fueron integrados a una base de datos predefinida en Excel (Microsoft, Inc). En el presente estudio se reportan los resultados con medidas de tendencia central (media, mediana) y sus respectivas medidas de dispersión (desviación estándar o rango). Para las variables nominales se emplearon el número total de pacientes y los porcentajes. Se utilizó el programa de análisis estadístico SPSS versión 18.0 (SPSS Inc. Chicago, IL, USA). 


\section{Resultados}

De enero de 2015 a diciembre de 2019 se encontraron 158 casos de pacientes operados por apendicitis aguda complicada mediante cirugía laparoscópica en nuestro centro, de los cuales 54 (34.2\%) se reportaron con base apendicular comprometida. De estos 54 casos que se incluyeron para el análisis, hubo un predominio del sexo masculino $(66.7 \%)$ y la edad media fue de 38.7 años (rango: 18-74). En cuanto al resto de las variables demográficas, se encontró una media de IMC de $27.4 \mathrm{~kg} / \mathrm{m}^{2}$, el $21.2 \%$ de los pacientes tenían alguna comorbilidad y el $55.5 \%$ fueron clasificados como ASA II. El resto de los datos se presentan en la tabla 1.

De los 54 pacientes con apendicitis aguda complicada, 37 (68.5\%) tenían la base apendicular necrosada y friable, 15 (27.8\%) tenían el ciego inflamado y friable más la base apendicular necrosada, y 2 (3.7\%) tenían la base apendicular perforada en continuidad con la transición con el ciego (Fig. 1).

En cuanto al tratamiento utilizado, en 31 pacientes (57.4\%) se realizó un nudo simple con sutura monofilamento no absorbible (polipropileno) con nudo deslizable según la técnica extracorpórea. En el resto de los pacientes $(42.6 \%)$ se realizó un punto transfictivo (sutura monofilamento no absorbible) con posterior invaginación del muñón apendicular (con sutura monofilamento absorbible). En 45 pacientes (83.3\%) se colocó un drenaje cerrado.

El tiempo quirúrgico medio fue de 113 minutos (rango: 55-195) y el sangrado transoperatorio medio se reportó de $89 \mathrm{ml}$ (rango: 10-360) (Tabla 2). Solo un paciente $(1.9 \%)$ requirió conversión a cirugía abierta (vía laparotomía exploradora).

En relación con las complicaciones posoperatorias, nueve pacientes desarrollaron absceso intraabdominal, de los cuales dos fueron resueltos mediante drenaje percutáneo guiado por tomografía más antibióticos, tres solo requirieron tratamiento antibiótico de amplio espectro y en cuatro fue necesario el drenaje quirúrgico mediante cirugía laparoscópica con lavado peritoneal y colocación de drenaje. Por último, cuatro pacientes presentaron íleo posoperatorio, el cual se resolvió con tratamiento médico (ayuno y sonda nasogástrica).

Por lo que concierne a la cantidad de fugas del muñón apendicular, así como de fistulas cecales, encontramos que, de los 54 pacientes, 1 (1.9\%) presentó a los 6 días de la cirugía salida de material fecal a través del drenaje. Esta paciente respondió con tratamiento conservador ambulatorio con antibiótico,

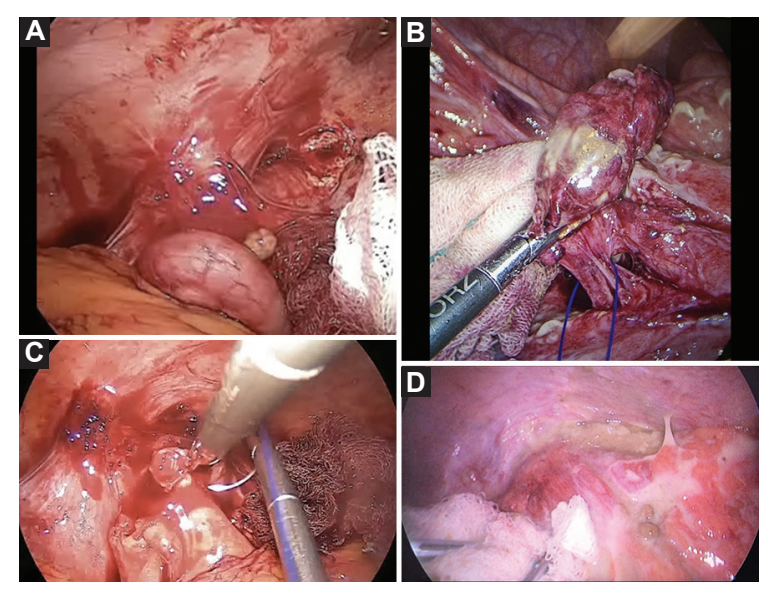

Figura 1. Apendicitis complicada con base apendicular comprometida. A: apendicitis con fecalito libre y adherencias. B: perforación de la base apendicular con ligadura de mesoapéndice. C: punto transfictivo a la base del apéndice perforada, con afección del ciego. D: apendicitis con fecalito libre y peritonitis purulenta generalizada.

Tabla 1. Datos sociodemográficos $(n=54)$

\begin{tabular}{lc}
\hline Variable & \\
\hline Edad media (min-max) & $38.7(18-74)$ \\
Sexo, n (\%) & $18(33.3)$ \\
Femenino & $36(66.7)$ \\
Masculino & $27.4(14.5-38.6)$ \\
IMC medio (min-max) & \\
Comorbilidad, n (\%) & $43(79.6)$ \\
Ninguna & $1(1.9)$ \\
DM2 + HAS & $4(7.4)$ \\
DM2 & $6(11.1)$ \\
HAS & \\
ASA, n (\%) & $52(96.3)$ \\
I-II & $2(3.7)$ \\
\hline
\end{tabular}

ASA: American Society of Anesthesiology; DM2: diabetes mellitus tipo 2; HAS: hipertensión arterial sistémica.

medidas dietéticas (dieta hiperproteica) y drenaje percutáneo a derivación. En cuanto hubo aclaramiento del gasto a través del drenaje se realizó una colotomografía, y al reportarse ausencia de fuga y colección, se retiró el drenaje.

Un paciente $(1.9 \%)$ con base apendicular necrosada y ciego friable (no perforado) desarrolló a los 3 días posoperatorios fuga del muñón apendicular. El diagnóstico se realizó debido a la presencia de material fecal a través del drenaje y las heridas. El paciente requirió reintervención mediante laparotomía exploradora, y debido a los hallazgos y las condiciones del paciente, fue 
Tabla 2. Resultados quirúrgicos $(n=54)$

\begin{tabular}{ll}
\hline Variable & \\
\hline Tiempo quirúrgico (min), media (min-max) & $113(55-195)$ \\
Sangrado transoperatorio (ml), media (min-max) & $89(10-360)$ \\
Conversión a cirugía abierta, n (\%) & $1(1.9)$ \\
Complicaciones posoperatorias, n (\%) & \\
Fuga del muñón & $1(1.9)$ \\
Fístula cecal & $1(1.9)$ \\
Complicaciones Clavien-Dindo, n (\%) & \\
Grado I & \\
Infección sitio quirúrgico superficial & $4(7.4)$ \\
Íleo & $3(5.6)$ \\
Grado II & \\
Íleo prolongado & $1(1.9)$ \\
Absceso, requirió antibiótico & $3(5.6)$ \\
Fístula cecal & $1(1.9)$ \\
Grado III-a & $2(3.7)$ \\
Absceso, requirió punción & $4(7.4)$ \\
Grado IIl-b & $1(1.9)$ \\
Absceso, requirió reintervención & \\
Reintervención por fuga & \\
\hline
\end{tabular}

necesario realizar una hemicolectomía derecha con ileostomía terminal.

Con respecto a las variables posoperatorias, en promedio se inició la dieta por vía oral a las 42.4 horas (rango: 24-168). El tiempo medio de estancia hospitalaria fue de 5.4 días (rango: 1-20). Se realizaron cinco reintervenciones: cuatro para drenaje de absceso intraabdominal y una por fuga del muñón apendicular. Durante los 90 días de seguimiento incluidos en el estudio, no se reportó ningún fallecimiento.

\section{Discusión}

En este estudio retrospectivo se encontró que, en los pacientes con apendicitis complicada con la base apendicular comprometida y tratamiento con ligadura simple o con punto invaginante, la cantidad de fugas y fístulas del muñón apendicular es muy baja $(<2 \%)$ y el resto de las complicaciones posoperatorias presentan frecuencias similares a las reportadas en otras series internacionales. La aportación de nuestro trabajo es que solo analizamos el subgrupo de pacientes con afección de la base apendicular. Cabe destacar que la mayoría de las series internacionales publicadas analizan las apendicitis complicadas sin discernir la localización anatómica de la perforación $6,8,14,15$.

Frazee y Bohannon ${ }^{14}$ fueron los primeros en concluir que la apendicectomía laparoscópica es segura y eficaz. No se ha demostrado claramente la superioridad de una técnica laparoscópica sobre las otras (por ejemplo, sutura, endo-loop, engrapadora), respecto al manejo de la base apendicular necrosada o perforada en la apendicitis aguda complicada. En la actualidad no existe un acuerdo mundial sobre algún método que sea considerado de referencia ${ }^{15,16}$.

La apendicitis complicada tiene una mayor tasa de complicaciones posoperatorias, con una incidencia que oscila entre el $3.0 \%$ y el $28.7 \%$. Estas incluyen infección del sitio quirúrgico (1.2-12.0\%), oclusión intestinal $(0-1.9 \%)$, abscesos intraabdominales (1.6$8.0 \%)$, fuga del muñón apendicular $(<1 \%)$ y fístulas estercoráceas $(<1 \%)$, entre otras ${ }^{17-19}$.

En 2014, Gomes et al. ${ }^{20}$ evaluaron la seguridad y la efectividad de la laparoscopía en el tratamiento de la apendicitis aguda complicada. De 154 pacientes con apendicitis aguda complicada, 14 (9.1\%) presentaron la base apendicular necrosada, y de estos, dos tuvieron infección del sitio quirúrgico. La conversión a cirugía abierta fue necesaria en ocho de los 154 pacientes $(5.19 \%)$, de los cuales cnco tenían la base apendicular necrosada (3.24\%). Los autores concluyeron que la base necrosada fue el factor más importante asociado con la conversión a cirugía abierta. En nuestro estudio, todos los pacientes se operaron con cirugía laparoscópica, solo un caso requirió conversión a cirugía abierta y encontramos un $16.7 \%$ de infección de sitio quirúrgico tipo órgano/espacio.

En un metaanálisis sobre el manejo óptimo del muñón en la apendicectomía laparoscópica, realizado por Antoniou, et al. $^{8}$ en 2017, no fue posible realizar un análisis de subgrupos según el grado de apendicitis y el involucro de la base apendicular, debido a que no se reportaba por separado en los estudios. Sin embargo, se sugiere que la apendicitis aguda con inflamación de la base apendicular puede ser tratada mejor con engrapadora o incluso con cirugía abierta.

En términos de nuestro objetivo principal, no existe información sobre la frecuencia de fuga o fístula del muñón apendicular en la apendicectomía laparoscópica con base apendicular comprometida en adultos. Lintula, et al. ${ }^{19}$ reportaron una frecuencia del $1 \%$ después de la apendicectomía laparoscópica en niños con apendicitis complicada, porcentaje similar al que encontramos en nuestra serie (1.9\%).

Nuestro estudio no está exento de limitaciones, de las que comentaremos las principales. En primer lugar, el hecho de que sea un estudio retrospectivo y en un solo centro hace que los resultados tengan riesgo de sesgo tanto de selección como de información. Podría 
parecer que son pocos pacientes para validar los resultados, pero debemos considerar que se incluyeron aquellos pacientes con apendicitis complicada en que el segmento afectado era la base del apéndice (34.2\% en nuestro estudio). Esto es importante porque el tercio medio del apéndice es el sitio que con más frecuencia se afecta o se perfora en un evento de apendicitis aguda complicada, por lo que la afección de la base apendicular es poco analizada de forma separada en los estudios. Otra limitación importante es que solo evaluamos la técnica con sutura del muñón apendicular, sin considerar otras técnicas como el uso de engrapadoras intestinales. El empleo de engrapadoras intestinales no es posible de forma inmediata en nuestro hospital. A pesar de esto, consideramos que la poca disponibilidad en nuestro medio de engrapadoras intestinales para realizar una apendicectomía hace que nuestros resultados con empleo de ligadura con sutura sean más representativos y generalizables a las condiciones de nuestro país.

Creemos que los resultados de este estudio pueden ser de interés para todos los cirujanos generales que se dedican a realizar apendicectomías laparoscópicas, dando certeza de que el empleo de la sutura o ligadura del muñón apendicular durante un procedimiento laparoscópico no se relaciona con un incremento de la frecuencia de fugas o fistulas fecales respecto a los datos reportados en la literatura médica internacional.

\section{Conclusiones}

De acuerdo con los resultados de este estudio, en los pacientes con apendicitis aguda complicada con base apendicular comprometida, el manejo laparoscópico con ligadura simple o con punto transfictivo e invaginante resulta en una baja frecuencia de fuga fecal del muñón apendicular y de fístulas cecales.

\section{Responsabilidades éticas}

Protección de personas y animales. Los autores declaran que para esta investigación no se han realizado experimentos en seres humanos ni en animales.

Confidencialidad de los datos. Los autores declaran que han seguido los protocolos de su centro de trabajo sobre la publicación de datos de pacientes.

Derecho a la privacidad y consentimiento informado. Los autores han obtenido el consentimiento informado de los pacientes y/o sujetos referidos en el artículo. Este documento obra en poder del autor de correspondencia.

\section{Financiamiento}

No se recibió ningún tipo de financiamiento para la realización de este estudio.

\section{Conflicto de intereses}

Los autores declaran no tener conflictos de intereses para la realización de este trabajo.

\section{Bibliografía}

1. Wilms IM, de Hoog DE, de Visser DC, Janzing HM. Appendectomy versus antibiotic treatment for acute appendicitis. Cochrane Database Syst Rev. 2011;(11):CD008359.

2. Buckius MT, McGrath B, Monk J, Grim R, Bell T, Ahuja V. Changing epidemiology of acute appendicitis in the United States: study period 1993-2008. J Surg Res. 2011;7:106.

3. Lee JH, Park YS, Choi JS. The epidemiology of appendicitis and appendectomy in South Korea: national registry data. J Epidemiol. 2010; 20:970105.

4. Gorter RR, Eker HH, Gorter-Stam MA, Abis GS, Acharya A, Ankersmit M, et al. Diagnosis and management of acute appendicitis. EAES Consensus Development Conference 2015. Surg Endosc. 2016;30:4668-90.

5. Schier F. Laparoscopic appendectomy with $1.7 \square \mathrm{mm}$ instruments. Pediatr Surg Int. 1998;14:142-3.

6. Di Saverio S, Podda M, De Simone B, Ceresoli M, Augustin G, Gori A, et al. Diagnosis and treatment of acute appendicitis: 2020 update of the WSES Jerusalem guidelines. World J Emerg Surg. 2020;15:27.

7. Gomes CA, Junior CS, de Peixoto RO, Netto JM, Gomes CC, Gomes FC. Appendiceal stump closure by metal endoclip in the management of complicated acute appendicitis. World J Emerg Surg. 2013;8:35.

8. Antoniou SA, Mavridis D, Hajibandeh S, Hajibandeh S, Antoniou GA, Gorter $\mathrm{R}$, et al. Optimal stump management in laparoscopic appendectomy: a network meta-analysis by the minimally invasive surgery synthesis of interventions and outcomes network. Surgery. 2017;162:994-1005.

9. Kiudelis M, Ignatavicius P, Zviniene K, Grizas S. Analysis of intracorporeal knotting with invaginating suture versus endoloops in appendiceal stump closure. Wideochir Inne Tech Malo Inwazyjne. 2013;8:69-73.

10. Ali N, Javaid A. El papel de la cecostomía con sonda en la prevención de abscesos y formación de fístulas después de la apendicectomía. Pak J Med Sci. 2005;21:285-8.

11. Vandenbroucke JP, von Elm E, Altman DG, Gøtzsche PC, Mulrow CD, Pocock SJ, et al. Strengthening the reporting of observational studies in epidemiology (STROBE): xplanation and elaboration. PLOS Med. 2007;4:e297.

12. Bolmers MDM, de Jonge J, van Rossem CC, van Geloven AAW, Bemelman WA; Snapshot Appendicitis Collaborative Study Group. Discrepancies between intraoperative and histological evaluation of the appendix in acute appendicitis. J Gastrointest Surg. 2020 Sep;24(9):2088-2095.

13. Clavien PA, Barkun J, de Oliveira ML, Vauthey JN, Dindo D, Schulick RD, et al. The Clavien-Dindo classification of surgical complications: five-year experience. Ann Surg. 2009;250:187-96.

14. Frazee RC, Bohannon WT. Laparoscopic appendectomy for complicated appendicitis. Arch Surg. 1996;131:509-13.

15. Kanona H, Al Samaraee A, Nice C, Bhattacharya V. Stump appendicitis: a review. Int J Surg. 2012;10:425-8.

16. Forgues D, Habbig S, Dillo AF, Kalfa N, Lopez M, Allal H, et al. Post-appendectomy intra-abdominal abscesses. Can they successfully be managed with the sole use of antibiotic therapy? Eur J Pediatr Surg. 2007;17:104-9.

17. Lau DHW, Yau KKK, Chung CC, Leung FCS, Tai YP, Li MKW. Comparison of needlescopic appendectomy versus conventional laparoscopic appendectomy: a randomized controlled trial. Surg Laparosc Endosc Percutan Techn. 2005;15:75-9.

18. Fleming FJ, Kim MJ, Messing S, Gunzler D, Salloum R, Monson JR. Balancing the risk of postoperative surgical infections: a multivariate analysis of factors associated with laparoscopic appendectomy from the NSQIP database. Ann Surg. 2010;252:895-900.

19. Lintula H, Kokki H, Vanamo K, Antila P, Eskelinen M. Laparoscopy in children with complicated appendicitis. J Pediatr Surg. 2002;37:1317-20.

20. Gomes CA, Junior CS, Costa Ede F, Alves P de A, de Faria CV, Cangussu IV, et al. Lessons learned with laparoscopic management of complicated grades of acute appendicitis. J Clin Med Res. 2014;6:261-6. 